\title{
Linfoma primario del sistema nervioso central en un paciente inmunocompetente
}

\author{
Primary central nervous system lymphoma in an immunocompetent
} patient

\author{
José Málaga-Zenteno ${ }^{1}$, Jersson Alonso Mamani-Quispe ${ }^{2, a}$, Mariela Fuentes Fuentes ${ }^{1}$, \\ José Alonso Suclla-Velásquez ${ }^{2, a}$, Julio Meza Aragón ${ }^{3}$ \\ ${ }^{1}$ Médico Asistente, Servicio de Hematología, Hospital Nacional Carlos Alberto Seguin Escobedo, EsSalud, Arequipa, Perú. \\ ${ }^{2}$ Estudiante de Medicina Humana, Centro de Investigación y Estudios Médicos (CIEM), Universidad Católica Santa María, Arequipa, Perú. \\ ${ }^{3}$ Médico Asistente, Servicio de Neurocirugía, Hospital Nacional Carlos Alberto Seguín Escobedo, EsSalud, Arequipa, Perú. \\ ${ }^{a}$ Sociedad Científica Médico Estudiantil Peruana (SOCIMEP).
}

\begin{abstract}
Resumen
El linfoma primario del sistema nervioso central (LPSNC) constituye el $2 \%$ de los linfomas extranodales y entre $0,3 \%$ y $1,5 \%$ de todas las neoplasias intracraneales en pacientes inmunocompetentes, siendo más frecuente a partir de los 60 años. Reportamos el caso de un paciente varón de 76 años, sin antecedentes médicos de importancia, que inició su enfermedad con inestabilidad en la marcha, dificultad para mover el hemicuerpo izquierdo, a predominio braquial, cefalea holocraneal y mareos. Ingresó a emergencia despierto, parcialmente desorientado, Glasgow 14 y ptosis palpebral derecha; además, hemiparesia e hipoestesia izquierda, a predominio crural. Por tomografía computarizada cerebral se evidenció imagen captadora de contraste en región frontoparietal derecha, intra y extra craneal, edema cerebral y desplazamiento de la línea media. Se realizó craneotomía y biopsia del tumor, diagnosticándose linfoma difuso de células grandes B del sistema nervioso central. Fue VIH negativo. Se descartó otro tumor primario y metástasis. Recibió esquema CHOD/BVAM y radioterapia. Evolucionó favorablemente. Según resonancia magnética cerebral postratamiento, desapareció el tumor.
\end{abstract}

Palabras clave: Linfoma, neoplasmas del sistema nervioso central, inmunocompetencia.

\section{Abstract}

Primary central nervous system lymphoma (PCNSL) constitutes $2 \%$ of extranodal lymphomas and $0,3 \%-1,5 \%$ of all intracranial neoplasms in immunocompetent patients, being more frequent after the sixth decade of life. We report a case of a 76 year-old man with no antecedents who started his disease with march instability, difficulty to move left side of his body with brachial predominance, holocraneal headache and dizziness. He arrived at emergency with Glasgow 14 and right eyelid ptosis. He had left sided hemiparesis and hypoesthesia with crural predominance and occasional disorientation. Brain computed tomography (CT) showed a contrastenhanced image in the right frontoparietal region with both extra- and intra-cranial components, cerebral edema and middle line deviation. Craniotomy and biopsy of the tumor resulted in diffuse large B cell lymphoma of the central nervous system. The patient was HIV negative. Other primary tumor and metastasis were discarded. He was treated with 'CHOD/BVAM' regimen and radiotherapy. He evolved favorably. No tumor was found in the post treatment brain MRI.

Key words: Lymphoma, central nervous system neoplasm, immunocompetence.

\section{An Fac med. 2012;73(3):245-9}

\section{INTRODUCCIÓN}

El linfoma primario del sistema nervioso central (LPSNC) representa el 2\% de los linfomas extranodales, cuya frecuencia se incrementa después de los 60 años ${ }^{(1)}$; además, constituye entre el $0,3 \%$ a $1,5 \%$ de todas la neoplasias intracraneales en pacientes inmunocompetentes ${ }^{(2)}$. En contraste, en pacientes con sida, este linfoma es diagnosticado entre 1,6 y 9\%, según diversos estudios ${ }^{(3,4)}$. Así, el único factor de riesgo establecido para desarrollar LPSNC es la inmunodeficiencia, sea esta congénita o adquirida.

Comunicamos el caso de un paciente con LPSNC difuso de células grandes B en un adulto mayor inmunocompeten- te, debido a su rara casuística y al éxito obtenido con el tratamiento empleado en un hospital regional del Perú.

\section{CASO CLÍNICO}

Varón de 76 años de edad, con tiempo de enfermedad de tres meses, de inicio insidioso, caracterizado por cefalea ho- 
locraneal que se exacerbaba con la tos, acompañada de mareos, a los que inicialmente no prestó importancia. Algunos días antes de su ingreso presentó inestabilidad en la marcha y dificultad para movilizar el hemicuerpo izquierdo, a predominio braquial. No tenía antecedentes patológicos de importancia.

$\mathrm{Al}$ ingreso al Servicio de Emergencia del Hospital Nacional Carlos Alberto Seguín Escobedo, EsSalud, Arequipa, Perú, el 14/01/2011, presentaba mal estado general, afebril, hemodinámicamente estable, no se palpaba adenopatías o visceromegalias; al examen neurológico se le encontró desorientado por momentos, Glasgow 14, ptosis palpebral derecha, hemiparesia e hipoestesia de hemicuerpo izquierdo a predominio crural y fondo de ojo normal. La tomografía computarizada (TC) cerebral reveló una imagen captadora de contraste en región frontoparietal derecha, intra y extra craneal (figura 1). La resonancia magnética nuclear (RMN) mostró al lóbulo temporal ocupado por masa hipointensa en T1 que realzó marcadamente luego de la aplicación de gadolinio, de 42 x 39 x $47 \mathrm{~mm}$, con edema perilesional, con efecto de masa sobre la línea media, colapsando el ventrículo lateral derecho y con borramiento de surcos cerebrales parietooccipitales (figura 2). Por el edema cerebral, recibió dexametasona y manitol vía endovenosa. Fue negativo para metástasis, según imagenología. Se descartó linfoma testicular con ecografía.

Se le realizó serología para VIH, resultando no reactivo; la biometría hemática fue normal, con hemoglobina $14,7 \mathrm{~g} / \mathrm{dL}$, plaquetas $348000 / \mathrm{mL}$, leucocitos $7280 / \mathrm{mL}$, abastonados $2 \%$, segmentados 67\%, neutrófilos 69\%, eosinófilos 3\%, monocitos $0 \%$, linfocitos $20 \%$, lactato deshidrogenasa elevada (463 u/L; normal 115 a $221 \mathrm{u} / \mathrm{L})$, beta 2 microglobulina $2200 \mathrm{mg} / \mathrm{dL}$, ácido úrico 2,7 mg/dL. Debido a hipertensión endocraneana, que aumentaba riesgo de enclavamiento cerebral por descompresión rápida, no se efectuó

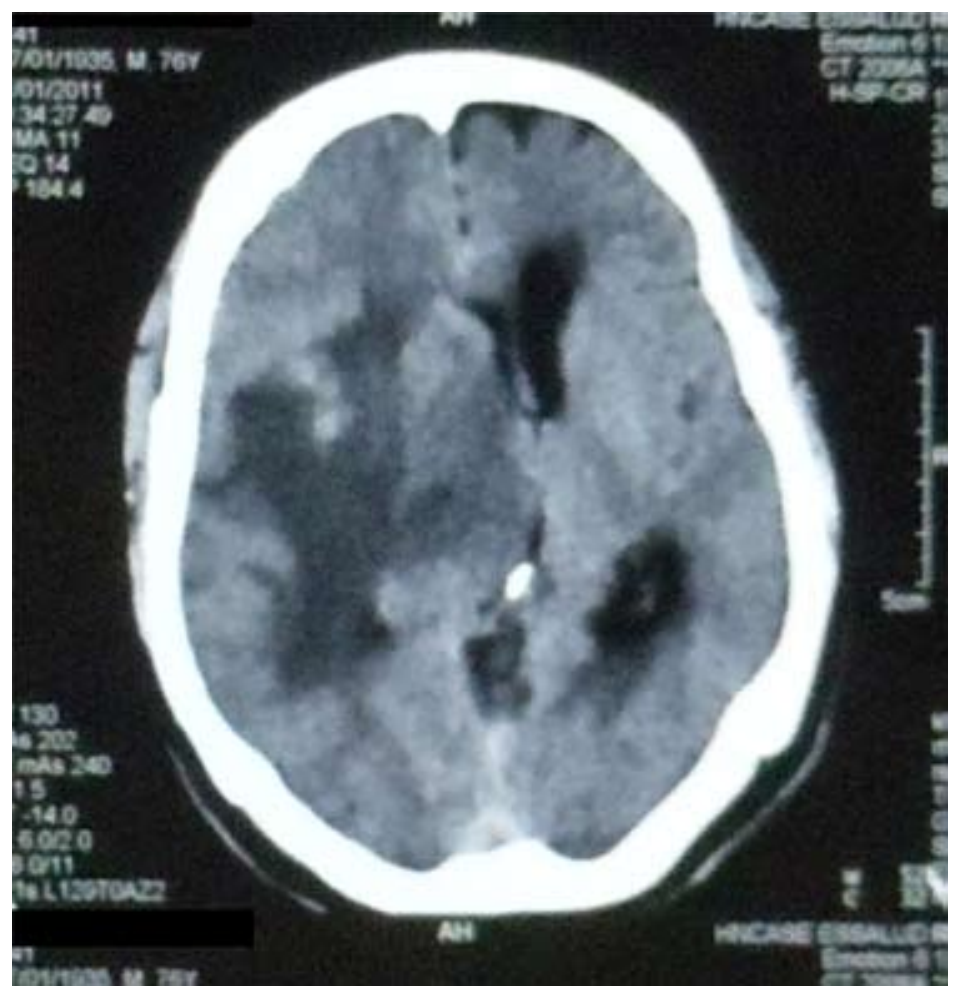

Figura 1. Tomografía cerebral revela imagen captadora de contraste en región frontoparietal derecha intra y extra craneal.

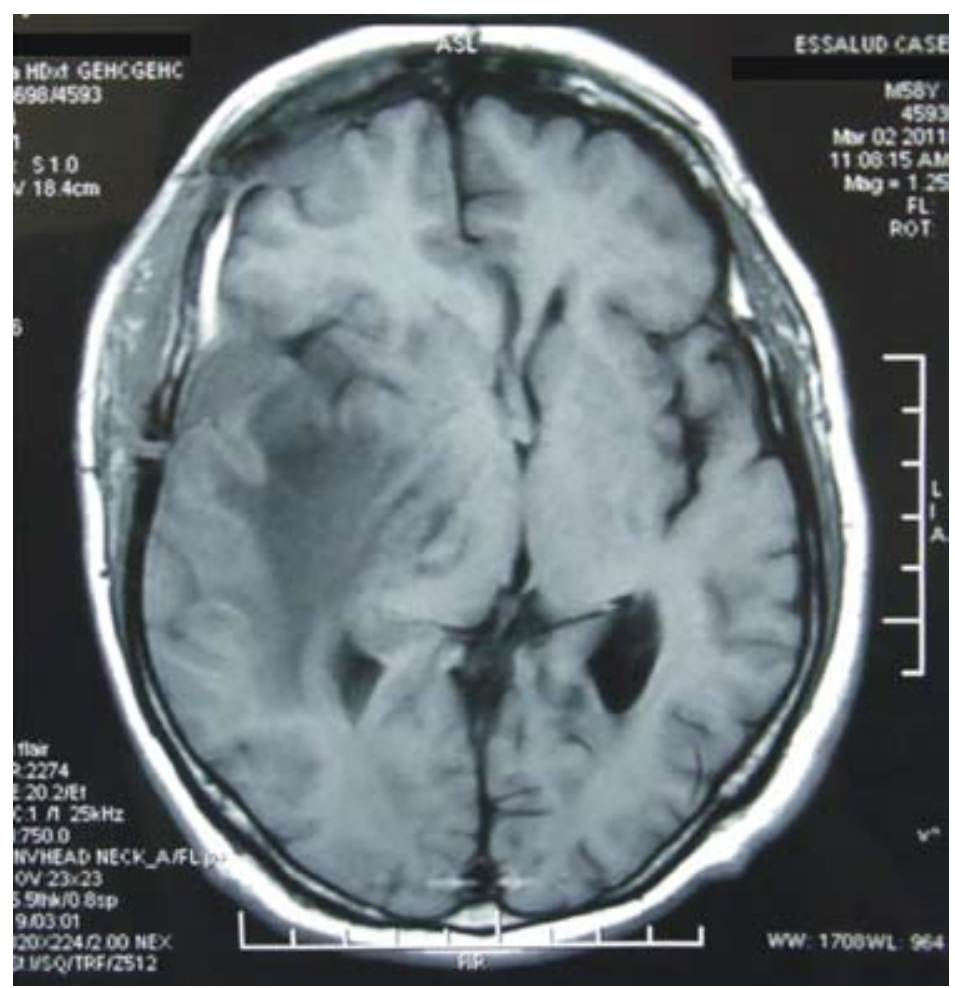

Figura 2. RMN craneoencefálica revela masa hipointensa en T1 en lóbulo temporal, con edema perilesional y efecto de masa sobre la línea media. 


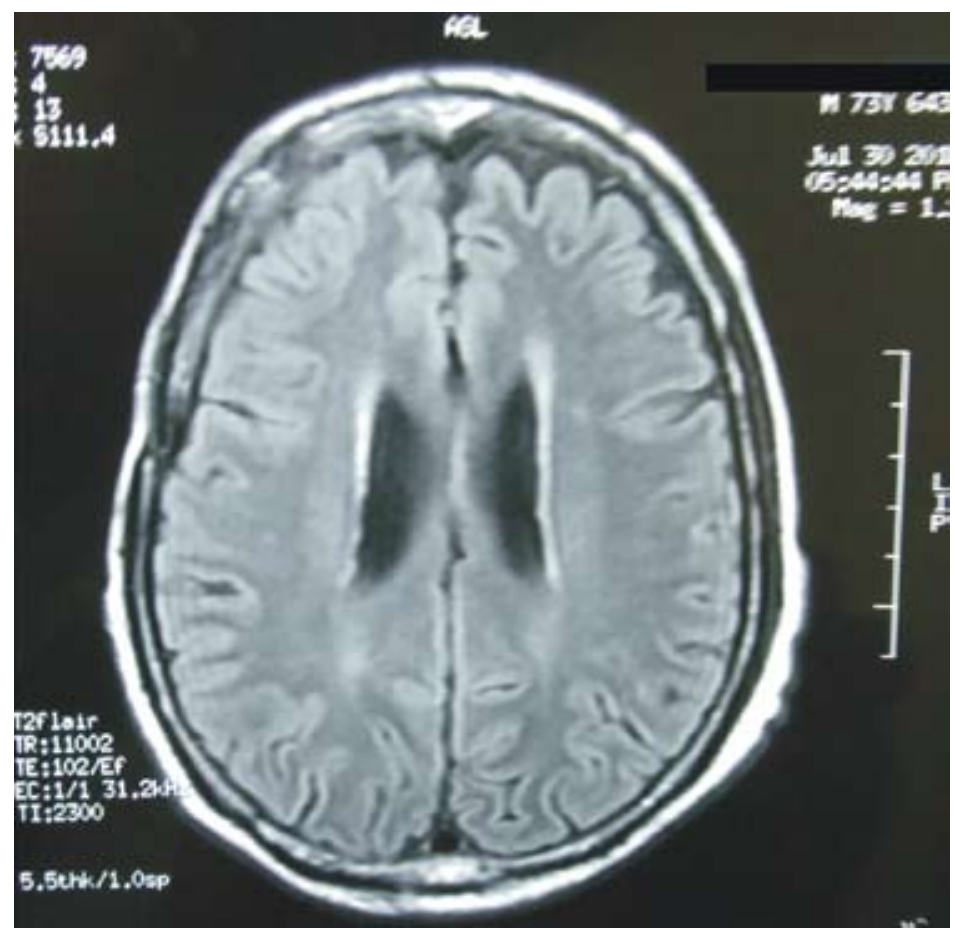

Figura 3. Resonancia magnética craneoencefálica postratamiento revela encefalomalacia temporal derecha. No se observa tumoración.

punción lumbar. El 25/01/2011 se le realizó craneotomía pterional y biopsia de tumoración, encontrándose que esta infiltraba músculo, hueso y duramadre, extendiéndose desde hueso frontal hasta el temporal, moderadamente vascularizada. El informe del estudio histopatológico fue linfoma difuso de células grandes B del sistema nervioso central. La inmunohistoquímica fue CD20 (+), CD10 (-), CD5 (+), BCL2 (+). En los estudios de aspirado y biopsia de médula ósea no se encontró infiltración linfomatosa.

Para el tratamiento se utilizó el esquema ciclofosfamida, adriamicina, vincristina, dexametasona, filgrastim, carmustina, metotrexato y citarabina (CHOD/BVAM) ${ }^{(5)}$ más radioterapia holocraneal 30 Gy en 15 sesiones, con refuerzo hasta llegar a $40 \mathrm{~Gy}$. Presentó neutropenia febril, por lo que se le administró filgrastim y antibioticoterapia, cediendo el cuadro. Luego del inicio del tratamiento refirió cefalea opresiva intensa que desapareció con el transcurrir de este. Presentó ocasionales episodios de excitación psicomotriz y desorientación, que desaparecieron posteriormente.

Evolucionó favorablemente, continuando con su tratamiento hasta completarlo, en julio del 2011, siendo evaluado entonces por el neurólogo, quien encontró al paciente despierto, orientado, con lenguaje coherente, sin signos de focalización. Psiquiatría lo evaluó, encontrándolo lúcido, orientado, colaborador, con cálculo alterado, sin adecuada interpretación de refranes; el paciente negó alteraciones perceptivas e ideas delusivas, siendo catalogado como un trastorno orgánico cerebral. El electroencefalograma postratamiento fue normal y la RMN craneoencefálica evidenció la desaparición del tumor, sin edema, área gliótica temporal derecha subcortical sin actividad, con área de encefalomalacia temporal derecha (figura 3). La sobrevida global hasta la actualidad es de un año, manteniendo su autonomía personal.

\section{DISCUSIÓN}

El linfoma primario del sistema nervioso central (LPSNC) de células B grandes, constituye un raro tipo de linfoma extranodal no Hodgkin, que se origina en cerebro, ojos, leptomeninges o médula espinal, sin evidencia de linfoma en otra localización en el momento del diagnóstico ${ }^{(6)}$. La incidencia de LPS$\mathrm{NC}$ en inmunocompetentes se incrementó de 4 casos por 10 millones, en 1982, a 28 y hasta 30 por 10 millones en $1989{ }^{(7)}$. Afecta más frecuentemente a los varones, con razón de 1,2:1 a 1,7:1 y la edad mediana al diagnóstico es 60 años ${ }^{(8)}$. Al respecto, nuestro paciente tenía 76 años al momento del diagnóstico.

Se desconoce por qué el LPSNC se desarrolla primariamente en un órgano que carece de sistema linfático ${ }^{(9)}$. Una hipótesis planteada considera que la clonación de células B malignas de origen sistémico podría desarrollarse en un órgano linfoide, expresando moléculas de adhesión específicas y selectivas que facilitan el establecimiento (homing) en las meninges, parénquima cerebral y compartimientos intraoculares (vítreo, retina y nervio óptico), donde mutaciones secundarias facilitarían su posterior proliferación ${ }^{(10)}$. Las quimioquinas CXCL12, CXCL13 atraen a las células B y han sido identificadas en las células B malignas del LPSNC. La coexpresión de CXCL9 y CXCL12 es un fuerte estímulo quimiotáctico para los linfocitos infiltrantes de tumores CXCR4+/CX$\mathrm{CR} 3+/ \mathrm{CD} 8+$ y para los linfocitos $\mathrm{B}$ malignos CXCR4+/CXCR3-, en el microambiente perivascular; también, hay un aumento del factor de crecimiento de células B: interleuquina 4 en el LPS. $\mathrm{NC}$, así como sobreexpresión de los protooncogenes c-myc y Pim-1 ${ }^{(8)}$. La forma activada del factor de transcripción STAT-6, un mediador de la expresión génica dependiente de la interleuquina 4 , fue encontrado dentro de las células tumorales y en el endotelio tumoral; esto se asoció con sobrevida corta en pacientes tratados con altas dosis de metotrexato ${ }^{(11)}$. Algunos compo- 
nentes de la matriz extracelular, como la osteopontina y la chitinasa 3-simil 1 son expresadas en niveles más altos en el LPSNC ${ }^{(12)}$. También, el gen regulador de la vía de señales de la proteína G (RGS)-13 ha sido encontrada expresándose dos veces más en el LPSNC que en el linfoma sistémico. Las proteínas RGS regulan negativamente las señales de las proteínas $G^{(13)}$. De otro lado, hay un aumento del microARN (miRNA miR-17-5p) en el LPSNC, en comparación con el linfoma ganglionar o testicular. Este micro ARN se cree que promueve el crecimiento tumoral vía la disminución de la expresión de los reguladores negativos de las vías de señales activadas por mitógenos ${ }^{(14)}$.

La forma clínica de aparición más frecuente del LPSNC es la focalidad neurológica $(70 \%)$, tal como sucedió en el presente caso, seguida por síntomas neuropsiquiátricos (43\%), hipertensión intracraneana (33\%) -que también presentó nuestro paciente-, crisis epilépticas (14\%) y síntomas oculares (4\%) como visión borrosa, disminución de la agudeza visual y ojo rojo doloroso. Los síntomas B son mucho más raros; en ellos, el compromiso intraocular es observado en 10 a $20 \%$ de pacientes al diagnóstico ${ }^{(8)}$. En los LPSNC, las lesiones son solitarias en $65 \%$ de los pacientes y multifocales en $35 \%{ }^{(15)}$; la localización más común se encuentra en la sustancia blanca supratentorial de los lóbulos frontoparietales ${ }^{(16)}$. En nuestro paciente, la afectación también fue supratentorial. Se localizan en los hemisferios (38\%), tálamo/ganglios basales $(16 \%)$, cuerpo calloso (14\%), región ventricular (12\%) y cerebelo $(9 \%){ }^{(9,17)}$. Nuestro paciente tuvo una lesión única que se originó en el parénquima cerebral e infiltró duramadre, hueso y músculo; esto está en relación a la naturaleza infiltrativa del tumor.

Por otro lado, el tiempo que transcurre desde los primeros síntomas hasta el diagnóstico varía desde unos pocos días hasta varios meses ${ }^{(18)}$; en nuestro caso, el tiempo de enfermedad fue tres meses.
Dentro de los estudios realizados, además de la anamnesis y el examen físico, tenemos la resonancia magnética, la tomografía, la evaluación oftalmológica, evaluación testicular, biopsias de tumoración y de médula ósea. De acuerdo a la imagenología, la mayoría de las lesiones son isodensas o hiperdensas en la TC (92\%) e isointensas o hipointensas en la RMN en T2, y casi todas las lesiones captan contraste homogéneamente, excepto tras la administración de corticoides, los cuales pueden ser oncolíticos, haciendo desaparecer el tumor (tumores fantasmas) ${ }^{(19)}$. En los inmunocompetentes se suele presentar lesión supratentorial única con realce homogéneo, lo que concuerda con nuestro caso. Los LPSNC generalmente se presentan como lesiones intraparenquimatosas, a diferencia de los linfomas secundarios que invaden las leptomeninges; mientras que en los inmunodeprimidos es usual la presencia de lesiones múltiples ${ }^{(20)}$.

El tratamiento se basa en la corticoterapia, quimioterapia y radioterapia, dado que son tumores sensibles a ellos que responden bien inicialmente, pero pueden recidivar incluso hasta el 90\% durante el primer año ${ }^{(9)}$, lo cual aún no ha ocurrido con nuestro paciente. La recidiva suele producirse dentro del SNC y en algunos casos a distancia. Si bien es cierto que en el LPSNC puede ocurrir remisión con corticoides, causando los tumores fantasmas (ghost tumors), que podrían dar dificultades en el estudio histopatológico; en nuestro paciente no ocurrió ello, pese a que se usó dexametasona antes de la biopsia (21). Existe consenso de que la combinación de quimioterapia con radioterapia es superior a la radioterapia sola ${ }^{(22)}$. Actualmente, este es uno de los métodos más comúnmente usados.

La relativa rareza del LPSNC evita la rápida realización de ensayos clínicos fase III; por lo tanto, nuestra dependencia en los resultados de ensayos clínicos fase II es crítica. Los fármacos quimioterápicos utilizados deben ser capaces de atravesar la barrera hematoencefálica, como la citarabina y el metotrexato.
Esta última droga es la más efectiva y ampliamente usada para LPSNC ${ }^{(23,24)}$. La adición de citarabina a metotrexato (ambos en altas dosis) provee un mejor resultado, con toxicidad aceptable comparada con la del tratamiento único con metotrexato a dosis altas ${ }^{(25)}$. En nuestro paciente usamos ambas drogas. En algunos protocolos de tratamiento se emplea quimioterapia con metotrexato intraventricular vía reservorio de Ommaya y no simplemente intratecal (mediante punción lumbar) ${ }^{(26)}$. La terapia intratecal podría ser realizada cuando no existe contraindicación y según el protocolo. En el presente caso se usó quimioterapia con esquema $\mathrm{CHOD} / \mathrm{BVAM}$ más radioterapia ${ }^{(5,27)}$. Con diferentes regímenes de quimioterapia y dosis de radiación, este manejo de primera línea ha sido evaluado en algunos estudios, obteniéndose tasas de remisión completa, entre $30 \%$ y $87 \%$, y tasas de sobrevida global a 5 años de $30 \%$ a $50 \%{ }^{(8)}$.

A pesar del tratamiento agresivo, la mayoría de pacientes con LPSNC empeora o recae y requiere una terapia de rescate. Aún para los pacientes que alcanzan respuesta completa, la mitad de ellos recaerá ${ }^{(24)}$. Dentro de las opciones para la recaída tenemos la quimioterapia sistémica con metotrexato y citarabina, la temozolamida ${ }^{(24)}$, el rituximab intraventricular, que pueden ser útiles en recidivas en pacientes que no los recibieron como tratamiento de primera línea ${ }^{(21)}$, así como la radioterapia ${ }^{(24)}$. El gran inconveniente de la terapia son las tasas altas de neurotoxicidad, especialmente en los mayores de 60 años (hasta $100 \%$ versus solo $30 \%$ de los menores de 60 años, al usar radioterapia) ${ }^{(28)}$. Se ha comprobado mayor deterioro cognitivo en los pacientes tratados con radioterapia y quimioterapia, que en aquellos tratados únicamente con quimioterapia. Nuestro paciente presentó cierto grado de neurotoxicidad, pero que no le impidió realizar sus actividades básicas personales.

Se ha encontrado cinco variables que afectan negativamente la sobrevida: edad mayor a 60 años, escala de 
calidad de vida en paciente oncológico (Eastern Cooperative Oncology Group ECOG) mayor a 1, niveles séricos de LDH elevados, hiperproteinorraquia y afectación de estructuras las cerebrales profundas (regiones periventriculares, ganglios basales, troncoencéfalo y cerebelo). Los pacientes con 0 a 1,2 a 3 , o 4 a 5 de esos factores de riesgo, tienen una sobrevida global a dos años de $80 \%$, $48 \%$ o $15 \%$, respectivamente ${ }^{(29)}$; en nuestro caso, el paciente presentó tres de estos factores. Además, se ha visto una asociación estadísticamente significativa entre la expresión tumoral del antígeno BCL-6 y una mayor sobrevida (mediana de 101 meses de sobrevida versus 14,7 meses en quienes no lo expresan) ${ }^{(19,30)}$; además, la expresión intratumoral de STAT-6 podría constituir un nuevo marcador para pronóstico en LPSNC ${ }^{(10,11)}$. El índice pronóstico internacional utilizado en los linfomas de células B grandes difusos de otras localizaciones usualmente no ayudan en el LPSNC.

\section{REFERENCIAS BIBLIOGRÁFICAS}

1. Kumar V, Abbas AK, Fausto N. En: Robbins and Cotran. Pathologic Basis of Disease. 2005;28:1413.

2. Alécio-Mattei T, Alécio-Mattei J, Aguiar PH, Ramina R. Primary central nervous system lymphomas in immunocompetent patients. Neurocirugia (Astur). 2006;17(1):46-53.

3. Rosenblum ML, Levy RM, Bredesen DE, Bredesen $\mathrm{DE}$, So YT, Wara W, Ziegler JL. Primary central nervous system lymphomas in patients with AIDS. Ann Neurol. 1988;23(Suppl):S13-S16.

4. Welch K, Finkbeiner W, Alpers CE. Autopsy findings in the acquired immune deficiency syndrome. JAMA. 1984;252(9):1152-9.

5. Bessell EM, Graus F, López-Guillermo A, Villá S, Verger E, Petit J, et al. CHOD/BVAM regimen plus radiotherapy in patients with primary CNS nonHodgkin's lymphoma. Int J Radiat Oncol Biol Phys. 2001;50(2):457-64.
6. Sierra del Rio M, Rousseau A, Soussain C, Ricard D, Hoang-Xuan K. Primary CNS Iymphoma in immunocompetent patients. The Oncologist. 2009;14(5):526-39.

7. Bellesso M, Bizzetto R, Pereira J, Beitler B, Pracchia LF, Chmome DAS. Linfoma primário do sistema nervoso central. Rev Bras Hematol Hemoter. 2008;30(1):54-60.

8. Ferreri AJ. How I treat primary central nervous system lymphoma. Blood. 2011;118(3):510-22.

9. Zazpe I, de Llano P, Gorosquieta A, Cabada T, Tuñón $T$, Vázquez A y col. Linfoma cerebral primario: revisión bibliográfica y experiencia en el Hospital de Navarra en los últimos 5 años (2000-2004). Anales Sis San Navarra. 2005;28(3):367-77.

10. Chan Ch. Primary vitroretinal lymphoma: a report from an International Primary Central Nervous System Lymphoma Collaborative Group Symposium. The Oncologist. 2011;16:1589-99.

11. Garcia-Manero G, Yang H, Kuang SQ, O'Brien $\mathrm{S}$, Thomas D, Kantarjian H. Epigenetics of acute lymphocytic leukemia. Semin Hematol. 2009;46:2432.

12. Rubenstein JL, Shen A, Batchelor TT, Kadoch C, Treseler P, Shuman MA. Differential gene expression in central nervous system lymphoma. Blood. 2009;113(1):266-7.

13. Khan RB. Is intrathecalmethotrexate necessary in the treatment of primary central nervous system lymphoma? J Neurooncol. 2002;58:175-8.

14. Cloonan N, Brown MK, Steptoe AL, Wani S, Chan WL, et al. The miR-17-5p microRNA is a key regulator of the G17S phase cell cycle transition. Genome Biol. 2008;9(8):R127.

15. Gerstner ER, Batchelor TT. Primary central nervous system lymphoma. Arch Neurol. 2010;67(3):291-7.

16. Slone HW, Blake JJ, Shah R, Guttikonda S, Bourekas EC. CT and MRI findings of intracranial lymphoma. AJR. 2005;184:1679-85.

17. Kuker W, Nagele T, Korfel A, Heckl S, Thiel E, Bamberg $\mathrm{M}$, et al. Primary central nervous system lymphomas (PCNSL): MRI features at presentation in 100 patients. J Neurooncol. 2005;72:169-77.

18. Alécio-Mattei T, Alécio-Mattei J, Aguiar PH, Ramina R. Primary central nervous system lymphomas in immunocompetent patients. Neurocirugia (Astur). 2006;17(1):46-53.

19. Coronel D, Gallardo C, Gamargo C. Linfoma primario del sistema nervioso central en una paciente inmunocompetente: Caso clínico. Rev Méd Chile. 2008;136:491-5.

20. Rademaker J. Hodgkin's and non-Hodgkin's lymphomas. Radiol Clin North Am. 2007;45(1):69-83.

21. Gerstner ER, Batchelor TT. Primary central nervous system lymphoma. Arch Neurol. 2010;67(3):291-7.

22. Ferreri AJ, Abrey LE, Blay JY, Borisch B, Hochman J, et al. Summary statement on primary central nervous system lymphomas from the Eighth International Conference on Malignant Lymphoma, Lugano, Switzerland, June 12 to 15, 2002. J ClinOncol. 2003;21(12):2407-14.

23. Batchelor T. Primary CNS Iymphoma. J Clin Oncol. 2006;24:1281-8.

24. NCCN Clinical Practice Guidelines In Oncology. Central Nervous System Cancers. Version 2.2011. NCCN .org

25. Ferreri AJ, Reni M, Foppoli M, Martelli M, Pangalis GA, et al. High-dose cytarabine plus high-dose methotrexate versus high-dose methotrexate alone in patients with primary CNS lymphoma: a randomised phase 2 trial. Lancet. 2009;374(9700):1512-20.

26. DeAngelis LM, Yahalom J, Thaler HT, Kher U. Combined modality therapy for primary CNS lymphomas. J Clin Oncol. 1992;10: 635-43.

27. Laack N, O'Neill BP, Ballman KV, O'Fallon JR, Carrero XW, et al. CHOD/BVAM chemotherapy and whole brain radiotherapy for newly diagnosed primary central nervous system lymphoma. Int J Radiat Oncol Biol Phys. 2011;81(2):476-82.

28. DeAngelis LM, Seiferheld W, Schold SC, Fisher B, Schultz CJ, Radiation Therapy Oncology Group Study 93-10. Combination chemotherapy and radiotherapy for primary central nervous system lymphoma: Radiation Therapy Oncology Group Study 93-10. J ClinOncol. 2002;20(24):4643-8.

29. Ferreri AJ, Blay JY, Reni M, Pasini F, Spina M, Ambrosetti A, et al. Prognostic scoring system for primary CNS lymphomas: the International Extranodal Lymphoma Study Group experience. J ClinOncol. 2003;21:266-72.

30. Braaten KM, Betensky RA, de Leval L, Okada Y, Hochberg FH, Louis DN, et al. BCL-6 expression predicts improved survival in patients with primary central nervous system Iymphoma. Clin Cancer Res. 2003;9:1063-9.

Artículo recibido el 31 de enero de 2012 y aceptado para publicación el 5 de abril de 2012.

Financiamiento:

No hubo fuente de financiamiento.

Conflicto de intereses:

En este trabajo no hay conflicto de intereses.

Correspondencia:

Dr. José Málaga-Zenteno

semazent@yahoo.es 